\title{
1 Access to household water quality information leads to safer water: a cluster randomized controlled trial in India
}

3

4 Mallory Trent ${ }^{1}$, Robert Dreibelbis ${ }^{2 *}$, Arjun Bir $^{3}$, Sachchida Nand Tripathi ${ }^{4}$, Pawan Labhasetwar $^{5}$, 5 Pranav Nagarnaik ${ }^{5}$, Andrew Loo ${ }^{3}$, Robert Bain ${ }^{6}$, Marc Jeuland $^{7}$, Joe Brown ${ }^{3 *}$

*Corresponding authors

1 Johns Hopkins University, Baltimore, MD, USA

2 London School of Hygiene and Tropical Medicine, 44 Keppel St, London WC1E 7HT, UK; robert.dreibelbis@1shtm.ac.uk, +44 (0) 2076368636

3 School of Civil and Environmental Engineering, Georgia Institute of Technology, 790 Atlantic Drive, Atlanta, GA 30332, USA; joe.brown@ @e.gatech.edu; +1 4043854579

4 Department of Civil Engineering \& Centre for Environmental Science and Engineering, Indian Institute of Technology - Kanpur, India

5 National Environmental Engineering Research Institute (NEERI), Nagpur, India

6 UNICEF - New York, NY, USA

7 Sanford School of Public Policy and Duke Global Health Institute, Duke University, Durham, NC, USA; Institute of Water Policy; National University of Singapore; Singapore 


\section{Abstract}

23 Household-specific feedback on the microbiological safety of drinking water may result in changes

24 to water management practices that reduce disease risk. We conducted a randomized, controlled 25 trial in India to determine if information on household drinking water quality could change 26 behavior and improve microbiological quality as indicated by E. coli counts. We randomly 27 assigned 589 participating households to one of three arms: (1) a messaging-only arm receiving 28 messaging on safe water management $(\mathrm{n}=237)$; (2) a standard testing arm receiving the same 29 messaging plus laboratory E. coli testing results specific to that household's drinking water $(\mathrm{n}=$ 30 173); and (3) a test kit arm receiving messaging plus low-cost $E$. coli tests that could be used at 31 the household's discretion $(\mathrm{n}=179)$. Self-reported water treatment increased significantly in both 32 the standard testing arm and the test kit arm between baseline and follow-up one month later. 33 Mean $\log _{10}$ E. coli counts per $100 \mathrm{ml}$ in household stored drinking water increased in the 34 messaging-only arm from 1.42 to 1.87 , while decreasing in the standard testing arm (1.38 to 0.89 , $3565 \%$ relative reduction) and the test kit arm (1.08 to $0.65,76 \%$ relative reduction). Findings 36 indicate that household-specific water quality information can improve both behaviors and 37 drinking water quality.

\section{Introduction}

Diarrheal disease is a leading cause of childhood mortality, resulting in an estimated 1.3 million 41 deaths in $2015^{1}$. The majority of diarrheal disease cases are attributable to fecal-oral transmission 42 of pathogens via widespread environmental contamination, with exposures linked to lack of 43 adequate sanitation at the household and community levels, poor hygiene, and unsafe food and 44 water $^{2,3}$. Although a substantial fraction of diarrheal deaths could potentially be averted by 
45 installing high-quality piped water supply systems where waterborne disease risks are greatest ${ }^{4,5}$, 46 infrastructure expansion is costly and time-consuming ${ }^{6}$. Approximately $39 \%$ of the world's

47 population still lacks access to a safely managed water supply ${ }^{7}$ and microbiologically unsafe 48 drinking water remains prevalent in low- and middle-income countries ${ }^{7-10}$.

Where safe water infrastructure is inadequate, communities and households can improve 50 or maintain water quality through household water management practices, including treating 51 drinking water and improving how household water is handled during transport and in the home.

52 Point-of-use drinking water treatment can improve microbiological quality and may also reduce 53 risk of enteric disease ${ }^{5,11}$. Storing drinking water in a container with a narrow opening, lid, or 54 spigot for dispensing reduces the risk of recontamination of water within the home $\mathrm{e}^{5,11,12}$.

Despite the evidence that better household water management can improve or maintain water quality and may improve health outcomes, adoption of new behaviors is often low ${ }^{13-16}$ and

57 challenging to sustain ${ }^{17}$. In part, this is due to the complex range of behavioral determinants that 58 inform water management practices, such as financial or time constraints, perceived convenience, 59 or taste preferences ${ }^{18-20}$.

Lack of knowledge about water quality and disease risk can be a barrier to the adoption of 61 improved household water management behaviors ${ }^{18,21-23}$. In low-income settings, water quality

62 testing may be limited and typically occurs far from the community ${ }^{21}$; as a result, individuals rarely 63 have access to timely and specific information on their own household or source water quality. 64 Providing water quality information directly to individuals, or enabling them to obtain it 65 themselves, may therefore help households overcome a key knowledge barrier. Such information 66 might also facilitate households' decision-making with respect to changing or improving their own 
67 water quality ${ }^{23}$. Direct provision of information is simple and less dependent on testing by target

68 beneficiaries, relative to provision of test kits. However, microbial water quality can be highly

69 variable over time and space (Supplemental Information), and so provision of test kits might better

70 allow beneficiaries to determine how best to maintain drinking water safety by allowing for

71 multiple points of testing as needed.

This paper presents the results of a cluster-randomized controlled trial (cRCT) of low-cost,

73 field-deployable microbiological water test kits distributed at the household level in the rural

74 Kanpur district of Uttar Pradesh, India. In India, where more than 100,000 children under 5 die of

75 diarrhea each year ${ }^{24}$, the proportion of the population with access to piped drinking water may be

76 as low as $24 \%^{2}$; piped water networks that are available are also at high risk of contamination due

77 to intermittent service 25,26 . We developed a standard information and education intervention

78 consisting of community meetings and household visits designed to improve knowledge and skill

79 related to managing and maintaining household water quality. This information was implemented

80 alone and in combination with interventions providing household-specific water quality

81 information. Water quality information included standard laboratory testing or the provision of

82 low-cost field-water quality test kits that could be used in the home.

We had three key objectives: 1) to determine whether provision of household-specific

84 water quality information alongside education on how to improve water quality leads to changes

85 in the microbiological contamination of stored household drinking water, as measured by E. coli counts; 2) to determine whether household specific water quality information would lead to 87 changes in key water management behaviors (storage, handling, and/or treatment); and 3) to determine whether household access to a novel low-cost and simple water quality test, distributed 89 to households to use on their own, results in differential improvements in the microbiological 
90 quality of household-stored drinking water and key water management behaviors compared with

91 controls receiving no specific water quality information.

\section{Methods and Materials}

\section{Study design}

94 The study design is based on standard approaches to cluster randomized controlled trials ${ }^{27}$. We 95 registered this trial before beginning field work, including pre-specification of hypotheses, 96 methods, and outcome measures (trial registration: NCT03021434, clinicaltrials.gov). The pre-

97 defined primary outcome variable was the arithmetic mean E. coli count ${ }^{28}$ from samples of 98 household drinking water collected at one unannounced visit 4 weeks post-baseline. Secondary 99 outcomes included self-reported household water treatment frequency and method, self-reported 100 primary drinking water source, self-reported water storage practices (e.g. keeping storage 101 container covered, using a storage container with a narrow opening), and availability of soap for 102 handwashing. Water storage practices and availability of soap were verified by direct observation. 103 Additional outcomes included self-reported prevalence of diarrhea, abdominal pain, and vomiting 104 (overall and among children under 5) in the 7 days prior to the survey ${ }^{29}$.

\section{Overview and sampling frame}

106 Our study took place in rural and peri-urban villages in the Kanpur district of Uttar Pradesh, India. 107 We chose this area due to limited access to safe drinking water ${ }^{30}$ and proximity to our laboratory 108 at the Indian Institute of Technology Kanpur (IIT-K). We obtained a list of all villages in the 109 Kanpur district from government census records ${ }^{30}$. We randomly selected sixty villages that had a 110 population between 100 and 1,000 households, did not receive chlorinated drinking water from 111 public utilities, and could be reached within two hours by car from IIT-K. Using simple 
112 randomization procedures, selected villages were allocated to one of two intervention arms or a

113 comparison arm, with weighting to increase comparison arm allocation for multiple hypothesis

114 testing. Because there was no available list of individuals or households within each village, we

115 utilized participatory mapping by village leaders to identify households with children under five.

116 We intentionally sampled households with children under five due to disproportionate diarrheal

117 disease burden within this population ${ }^{1}$. Within each village catchment area, we randomly selected 118 ten of these identified households.

After a given household was recruited, trained data collectors reviewed a participant 120 information sheet with the respondent, which explained the project's overall objectives, duration

121 of the study, and general study procedures. We obtained written informed consent from all 122 participants prior to data collection activities, consistent with study approvals from institutional 123 review boards at the London School of Hygiene and Tropical Medicine (Ref. No.:11920) and IIT124 K (IITK/IEC/2016-17 II/4).

\section{Intervention}

126 The intervention consisted of three components: 1) a community education session combined with 127 information on household water management; 2) household education on household drinking 128 water management; and 3) provision of information about household-specific water quality. 129 Participants received household specific water quality data in one of two ways depending on study 130 arm. The messaging-only arm received only the first two components and received no information 131 on their household's stored water quality. For the purposes of this study, this messaging-only arm 132 serves as the comparison (or control) arm for the study. In the standard testing arm, trained data 133 collectors analyzed household water quality data in a laboratory by membrane filtration for E. coli. 134 Data collectors then returned to households and informed them whether or not their water was 
135 contaminated. In the test kit arm, each household was provided with ten water testing kits yielding 136 semi-quantitative results for $E$. coli, which they were instructed to use at their discretion. All 137 households received three visits during the intervention (two at baseline and one unannounced 138 follow up visit four weeks later), as explained in additional detail below.

The E. coli test kit used by participants in this trial was developed in prior pilot testing in 140 India [Supporting Information]. The semi-quantitative test uses the open-source Aquatest (AT) 141 broth medium ${ }^{31}$ with a resorufin methyl ester chromogen ${ }^{32}$ (Biosynth AG, Switzerland) and 142 ambient temperature incubation ${ }^{33}$ for 48 hours following sample collection. Briefly, water samples 143 are measured to $10 \mathrm{ml}$ and $100 \mathrm{ml}$ volumes using single-use volumetric cylinders that also serve 144 as packaging. These volumes are added to sealable bags containing pre-measured AT medium. A 145 color change from yellow-beige to pink-red indicates the presence of E. coli, and the combination 146 of the two bags is used to interpret the final test result. Results can be interpreted as $<1$ E. coli per $147100 \mathrm{ml}$ (both bags negative, "safe”); 1 - 9 E. coli per $100 \mathrm{ml}$ (large bag positive, small bag negative, 148 "unsafe - low risk"); or $\geq 10$ E. coli per $100 \mathrm{ml}$ (small bag positive or both bags positive, "unsafe $149-$ medium to high risk"). Users were asked to interpret test results themselves at the end of the 48150 hour ambient temperature incubation period using a graphic interpretation card that was provided 151 as part of the test. Illustrated step-by-step test instructions were also included with each kit 152 (Supporting Information). All product labeling and documentation was in Hindi. Project 153 enumerators spent approximately 5-10 minutes training each head of household (in Hindi) on use 154 of the test by carefully reviewing each step in the process and explaining how to interpret the test 155 results. Because E. coli counts in water can be highly variable (Supporting Information), even 156 within the same household and on the same day, multiple tests are often recommended to estimate 
157 water quality. In this trial, participants were supplied with 10 test kits and encouraged to use them 158 for multiple sources or at multiple time points, at the participant's discretion.

The intervention design was informed by the 'extended parallel processing model 160 (EPPM) ${ }^{, 34}$, a model which describes how behaviors are shaped by two broad determinants: 161 efficacy beliefs and perceived threat. All participating villages received the community education 162 and generalized household water management messaging. We designed household materials and 163 information sessions (Supporting Information) to target efficacy beliefs by demonstrating methods 164 that individuals can use to improve and maintain the microbiological quality of their water, 165 including storing water to avoid contact with hands, boiling water, and hand washing with soap. 166 Water quality test results and water quality test kits are assumed to target perceived susceptibility 167 to water contamination by providing households with specific information about the quality of 168 water in their own households. We tailored the information to be appropriate for local 169 circumstances and resources; focusing education materials and information sessions on behaviors 170 with low resource requirements for the household (e.g. boiling drinking water using readily 171 available biomass, handwashing with soap, storing water in a covered container), rather than cost172 intensive behaviors (e.g. switching to treated bottled water, purchasing commercial water filters, 173 using bleach/chlorine tablets). Project staff scheduled village information sessions in advance, and village leaders 175 promoted the sessions among mothers and female heads of households, since they are typically 176 responsible for management of household drinking water ${ }^{35}$. The session consisted of a short, 1517730 minute presentation on waterborne disease, water management, and strategies for improving 178 water quality in the home. Village information sessions were designed to be relatively informal, 179 and study staff encouraged questions and discussion among participants. Although the information 

193 hours.

session was mainly targeted to adult women, children often attended since the presentations typically took place in school buildings.

Following the community information session, data collection staff met with village leaders to define the boundaries of the village via participatory mapping and to identify households having at least one child under the age of five. From this, we recruited a random sample of ten households in the community to be part of the trial. To minimize bias, recruitment was not restricted to those that attended the community information session. Trained field staff visited the homes of all households recruited. While there, the enumerator spent 10-15 minutes reviewing water quality and management information with the head of household and other family members prior to completing the survey and water sample collection. All households were informed that data collectors would be returning after 72 hours and again after approximately one month for a follow up visit. Households in the test kit arm were also given a test kit and instructed on how to use it. Project staff instructed them to use this test on their household drinking water within 24

Following baseline data collection, all households were revisited within 72 hours. For households in the messaging-only arm, enumerators reviewed the water quality and management information again but did not provide any water quality results. For households in the standard testing arm, the data collector reviewed with the head of household whether or not their water had been found to be contaminated and reviewed the water quality and management information. For households in the test kit arm, the enumerator reviewed the results of the test and provided an additional nine test kits, which they were instructed to use on their household drinking water at their discretion. They also reviewed the water quality and management information. 
All households received an unannounced follow up visit approximately four weeks after the initial baseline visit. After completing data collection activities, data collection staff informed households in the messaging-only arm whether their drinking water sample from the baseline visit was contaminated.

\section{Sample Size}

We used standard formulae developed for statistical analysis of multi-intervention randomized controlled trials $\mathrm{s}^{27,36-38}$, accounting for clustering in the comparison of means for continuous outcomes. A coefficient of variation $(\mathrm{k})$ of 0.3 was used for sample size calculations based on previous microbial data collected during pilot work in Maharashtra (Supporting Information). We weighted arm allocation to minimize variance for multiple hypothesis testing ${ }^{38}$, resulting in a 4:3:3 control:intervention ratio in cluster allocation.

Sample size calculations assumed a mean baseline $E$. coli count of $85 \mathrm{cfu} / 100 \mathrm{ml}$ with a standard deviation of 290 as a conservative estimate based on previous systematic sampling of small, rural water supplies and stored drinking water in Maharashtra (Supporting Information). To allow a minimum detectable effect size (MDES) of $0.5 \log _{10}$ on the continuous outcome of $E$. coli $\mathrm{cfu} / 100$ $\mathrm{ml}$ at $80 \%$ power, we calculated that the sample would require 10 households per cluster, spread among 20 control villages and 15 intervention villages per arm (500 households). This sample size was determined to be sufficient for detecting the MDES between each intervention arm and the messaging-only control but was not intended to detect for differences between the intervention groups. We recruited an additional 10 villages ( 4 control villages, 3 per intervention arm) to allow for additional qualitative data collection following the conclusion of end line data collection, resulting in a total sample size of 60 villages and 589 households (Figure 1), which also allowed for some loss to follow-up among participants. 
Figure 1. CONSORT ${ }^{39}$ diagram describing the cluster randomized controlled trial design of the study

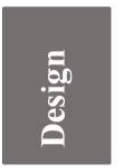

A cluster randomized controlled trial of low-cost microbiological water test kits
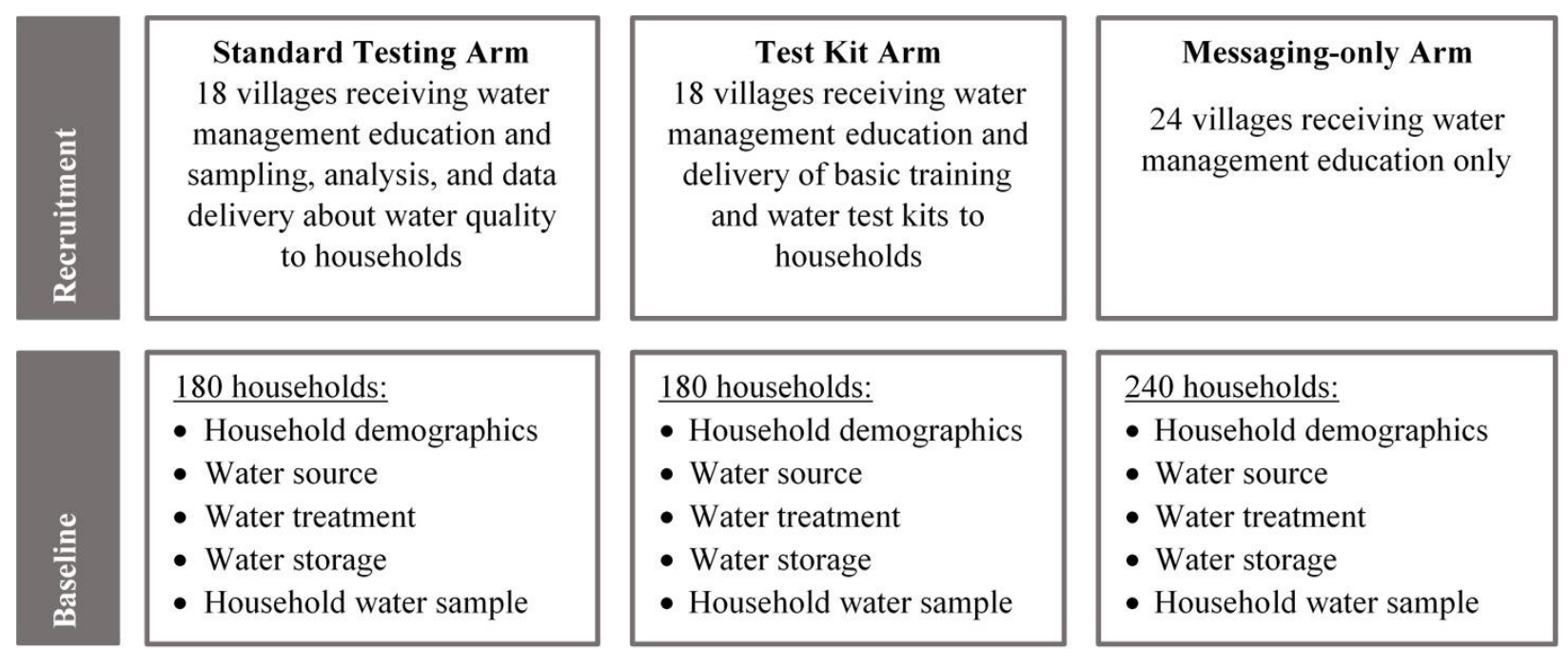

180 households:

- Household demographics

- Water source

- Water treatment

- Water storage

- Household water sample
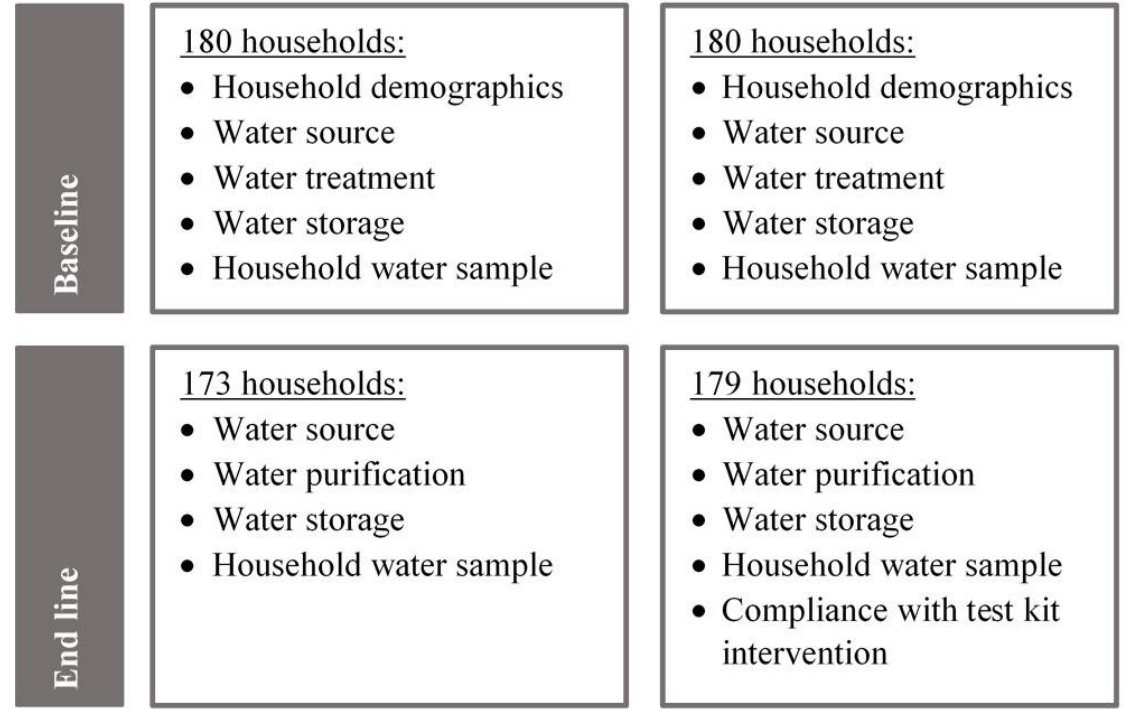

240 households:

- Household demographics

- Water source

- Water treatment

- Water storage

- Household water sample

179 households:

- Water source

- Water purification

- Water storage

- Household water sample

- Compliance with test kit intervention

Difference-in-difference analysis of outcome measures, controlling for demographic factors such as level of education, socio-economic status, and occupation

228 Data collection took place between March and May of 2017, during the dry season in Uttar

229 Pradesh. All of these activities were administered during unannounced baseline and follow up 
234 source, treatment, and storage of drinking water currently stored in the household. The respondent

235 provided details on children under the age of five, including name, age, and diarrhea episodes in

236 the previous week. Structured observations of household water storage, water treatment materials,

237 and handwashing materials were included in the survey questionnaire. Data collectors conducted

238 the surveys in Hindi and recorded responses electronically using mWater (http://www.mwater.co/)

239 software installed on smartphones. Phones were synched daily to an online database.

At both baseline and the follow up, trained data collectors collected a $330 \mathrm{ml}$ sample of

241 household drinking water for analysis. To collect the sample, we asked study participants to fill

242 the sample container (treated with sodium thiosulfate) as if it was a drinking cup for a child living

243 in the household. Samples were kept on ice in a cooler until delivery to the laboratory and thereafter

244 stored at $4^{\circ} \mathrm{C}$ until processing. All samples were processed within eight hours of the time of 245 sampling. E. coli in samples were enumerated by membrane filtration and incubation on selective 246 media consistent with EPA Method $1604^{40}$, though with membrane filters incubated on Compact 247 Dry EC plates (Hardy Diagnostics, Santa Maria, California) re-hydrated with $1 \mathrm{ml}$ of sample water.

248 Samples were processed and incubated for 24 hours at $35^{\circ} \mathrm{C}$; colony forming units (cfu) were 249 counted and reported as mean cfu per $100 \mathrm{ml}$ sample. For statistical purposes, if zero colony250 forming units were observed on the plate, we assigned a value of $0.5^{41}$. Likewise, if colonies were 251 too numerous to count reliably, we assigned a value of 200 as a conservative estimate of the upper 252 detection limit.

\section{Statistical analysis}

E. coli concentrations were log-transformed prior to analysis. Differences in baseline 255 household characteristics and E. coli concentration between study arms were assessed using linear 256 and logistic regression models, accounting for clustering at the village level. To determine whether 
257 there were significant differences in primary and secondary outcome measures between the 258 intervention arms and comparison arm, we utilized a difference-in-differences (DiD) approach ${ }^{42}$. 259 This method estimates the effect of specific interventions while adjusting for any inherent 260 differences between the intervention and control groups at baseline that may influence results. We 261 completed analysis in Stata v14 (College Station, Texas) using the 'xtgee' command, where 262 difference-in-difference analysis is estimated as the interaction term of the data collection round 263 (baseline vs. end line) and intervention arm (standard testing or test kit vs. messaging-only). 264 Generalized estimating equations (GEE) with robust variance estimation accounted for 265 correlations due to clustering ${ }^{43}$. The GEE model assumes that missing observations are Missing 266 Completely at Random (MCAR), but re-estimation using only the sample of households present 267 over the study duration yielded nearly identical results ${ }^{44}$. All analyses were adjusted for education 268 level completed and below poverty line status, which varied significantly across study groups.

269 To determine whether the presence of a contamination signal resulted in greater 270 improvements in water quality or reported water management behaviors, we performed a 271 difference-in-difference analysis within each of the two intervention arms comparing households 272 that received a contamination signal versus households that did not. However, this analysis was 273 below the unit of randomization, and therefore results should be interpreted with caution. 
Table 1. Selected baseline household characteristics and outcomes by treatment arm

\begin{tabular}{|c|c|c|c|c|c|}
\hline & $\begin{array}{c}\text { Messaging- } \\
\text { only }(\mathrm{N}=237)\end{array}$ & $\begin{array}{c}\text { Standard } \\
\text { Testing } \\
(\mathbf{N}=173)\end{array}$ & $\begin{array}{l}\text { Test Kit } \\
(\mathrm{N}=179)\end{array}$ & $\begin{array}{c}\text { Total } \\
(\mathbf{N}=\mathbf{5 8 9})\end{array}$ & p-value ${ }^{1}$ \\
\hline \multicolumn{6}{|l|}{ Demographic characteristics } \\
\hline Mean number of household members (SD) & $8.0(3.7)$ & $7.9(5.5)$ & $7.6(3.6)$ & $7.8(4.3)$ & 0.64 \\
\hline $\begin{array}{l}\text { Mean number of children under } 5 \text { per } \\
\text { household (SD) }\end{array}$ & $1.5(0.8)$ & $1.5(0.7)$ & $1.4(0.6)$ & $1.4(0.7)$ & 0.35 \\
\hline $\begin{array}{l}\text { Proportion of respondents that completed } \\
\text { secondary school (SE) }\end{array}$ & $0.51(0.03)$ & $0.58(0.04)$ & $0.41(0.04)$ & $0.50(0.02)$ & 0.03 \\
\hline $\begin{array}{l}\text { Proportion of households living below } \\
\text { poverty line (receives Antyodaya/BPL } \\
\text { ration card) (SE) }\end{array}$ & $0.33(0.03)$ & $0.45(0.04)$ & $0.55(0.04)$ & $0.43(0.02)$ & 0.03 \\
\hline \multicolumn{6}{|l|}{ Water quality, source, and treatment } \\
\hline $\begin{array}{l}\text { Proportion reporting primarily using } \\
\text { protected dug well to obtain water (SE) }\end{array}$ & $0.86(0.02)$ & $0.77(0.03)$ & $0.88(0.02)$ & $0.82(0.01)$ & 0.16 \\
\hline $\begin{array}{l}\text { Proportion reporting ever treating drinking } \\
\text { water, all methods (SE) }\end{array}$ & $0.01(0.01)$ & $0.05(0.02)$ & $0.04(0.01)$ & $0.03(0.01)$ & 0.07 \\
\hline $\begin{array}{l}\text { Mean } \log _{10} E \text {. coli cfu/100 } \mathrm{ml} \text { of household } \\
\text { drinking water }\end{array}$ & $1.42(1.76)$ & $1.38(1.57)$ & $1.09(1.54)$ & $1.31(1.64)$ & 0.29 \\
\hline \multicolumn{6}{|l|}{ Health outcomes } \\
\hline $\begin{array}{l}\text { Proportion of households with at least one } \\
\text { diarrhea case in the } 7 \text { days prior to } \\
\text { survey (SE) }\end{array}$ & $0.08(0.02)$ & $0.12(0.02)$ & $0.07(0.02)$ & $0.09(0.01)$ & 0.38 \\
\hline $\begin{array}{l}\text { Proportion of households with at least one } \\
\text { diarrhea case in a child under } 5 \text { in the } 7 \\
\text { days prior to survey (SE) }\end{array}$ & $0.04(0.01)$ & $0.09(0.02)$ & $0.04(0.02)$ & $0.06(0.01)$ & 0.10 \\
\hline
\end{tabular}

278 Household characteristics

279 Table 1 summarizes baseline statistics for the three study cohorts, as well as for the total sample.

280 The average household in this sample consisted of 7.9 members, including 1.4 children less than 2815 years old. Household composition did not vary significantly across the three study cohorts $(\mathrm{p}=$ $2820.64, \mathrm{p}=0.35)$. Approximately $50 \%$ of respondents completed secondary school, although this was lower in the test kit arm $(\mathrm{p}=0.03) .43 \%$ of households reported receiving a BPL (below poverty line) ration card from the government, with fewer households in the messaging-only arm $(33 \%)$ compared to the standard testing and test kit arms (45\% and $55 \%$ respectively) $(\mathrm{p}=0.03)$. 
288 reported obtaining drinking water from either a private or public protected dug well, which is

289 considered an "improved" water source. Water treatment, by any method, was uncommon among

290 all cohorts, with only 3\% of households reporting ever treating their water. The proportion of

291 households that reported treating their drinking water did not vary significantly across study arms

$292(\mathrm{p}=0.07)$. Of these households, participants reported boiling and using a commercial water filter

293 as methods of treatment. An estimated $8 \%$ of households reported that at least one member of the

294 household had experienced diarrhea in the 7 days preceding the survey, which was consistent

295 across study arms $(\mathrm{p}=0.38)$. An estimated $6 \%$ of households reported diarrhea in a child under 5

296 in the 7 days prior to the survey, which did not vary significantly across study arms $(\mathrm{p}=0.19$.

Only $11(1.8 \%)$ households were unavailable at the time of the one-month follow-up visit.

298 Additionally, $4.5 \%$ of households had incomplete E. coli concentration data, since some 299 households did not have stored drinking water available at the time of sampling. To determine 300 whether this affected the GEE results, we re-estimated the models with only households with 301 complete data. The results were nearly identical to those obtained using the full sample (results 302 not shown).

\section{Primary and Secondary Outcomes}

304 Water Quality Results

305 We collected a 1,160 water samples in total across all study arms and both data collection

306 rounds. Approximately $18 \%$ of samples fell below the detection limit ( $<1 \mathrm{cfu} / 100 \mathrm{ml})$ and $5 \%$ of 307 samples were above the detection limit ( $\geq 200 \mathrm{cfu} / 100 \mathrm{ml})$; the proportion of values censored at 3080 and 200 did not vary significantly across treatment arms $(\mathrm{p}=0.16$ and $\mathrm{p}=0.10$, respectively). 
Figure 2 presents the distribution of $E$. coli concentrations at baseline and one-month

311 follow up, based on commonly used $\log _{10}$ levels indicating potential risk ${ }^{45}$, by study arm. Table 2

312 outlines the changes in water quality and self-reported water management behaviors between

313 baseline and end line one month later, including differences in changes among treatment cohorts

314 and the messaging-only cohort. In the messaging-only arm, water quality did not improve: $\log _{10}$

315 mean E. coli $\mathrm{cfu} / 100 \mathrm{ml}$ increased from 1.42 to $1.87(8.4 \%)$ or from an arithmetic mean of 23

$316 \mathrm{cfu} / 100 \mathrm{ml}(95 \% \mathrm{CI} 16-30 \mathrm{cfu} / 100 \mathrm{ml})$ to $25 \mathrm{cfu} / 100 \mathrm{ml}(95 \% \mathrm{CI} 19-32 \mathrm{cfu} / 100 \mathrm{ml})$. In the

317 standard testing arm, water quality improved significantly between baseline and follow up. $\log _{10}$

318 mean E. coli $\mathrm{cfu} / 100 \mathrm{ml}$ decreased from 1.38 to $0.89(57 \%)$, which corresponds to a $0.94 \log _{10} \mathrm{cfu}$

319 / $100 \mathrm{ml}(65 \%)$ reduction compared to the messaging-only arm ( $<<0.01)$, after adjusting for

320 baseline differences; this corresponds to a decline from an arithmetic mean E. coli count of 16

$321 \mathrm{cfu} / 100 \mathrm{ml}(95 \% \mathrm{CI} 10-23 \mathrm{cfu} / 100 \mathrm{ml})$ at baseline to $7 \mathrm{cfu} / 100 \mathrm{ml}(95 \% \mathrm{CI} 4-10 \mathrm{cfu} / 100 \mathrm{ml})$ at

322 end line. As in the standard testing arm, we observed a significant improvement in water quality

323 in the test kit arm. $\log _{10}$ mean E. coli cfu/100 ml decreased from 1.09 to 0.65 (68\%), which

324 corresponds to a $0.84 \log _{10}(76 \%)$ reduction compared to the messaging-only arm $(\mathrm{p}<0.01)$, after

325 adjustment for baseline differences. This represents a decrease from an arithmetic mean E. coli

326 count of $12 \mathrm{cfu} / 100 \mathrm{ml}(95 \%$ CI $7-16 \mathrm{cfu} / 100 \mathrm{ml})$ at baseline to $4 \mathrm{cfu} / 100 \mathrm{ml}(95 \%$ CI 3 - 5

$327 \mathrm{cfu} / 100 \mathrm{ml})$ at end line in the test kit arm. 
Figure 2. Distribution of categorical ${ }^{45} E$. coli concentrations in household stored drinking water samples by surveillance point and study arm.

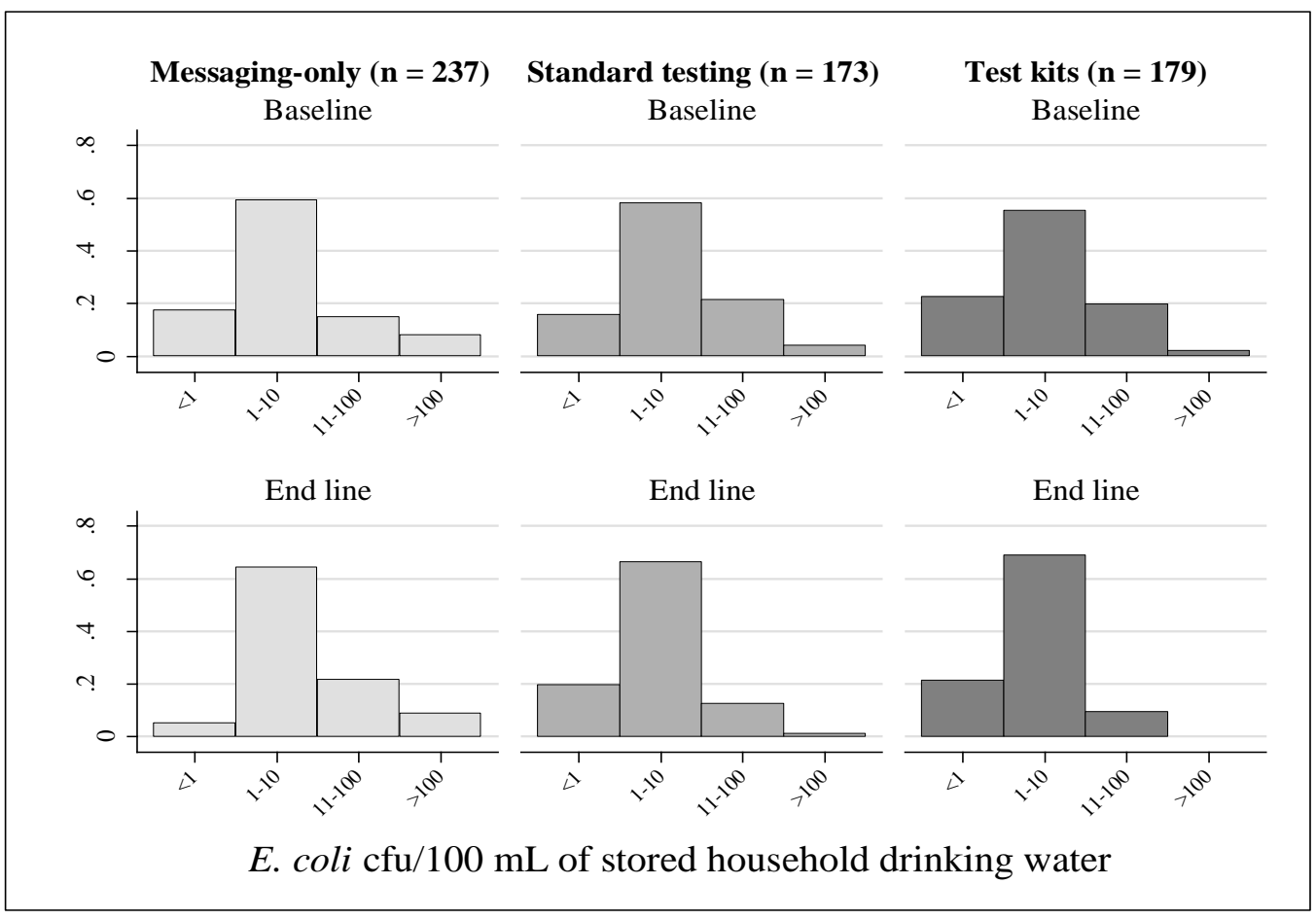

\section{Behavioral Outcomes}

Measured improvements in water quality align with changes in self-reported water treatment behaviors. In all study arms, there was an increase in the proportion of households that reported boiling drinking water in the previous two weeks. In the messaging-only arm, reported boiling in the previous two weeks increased from $<0.01$ to 0.04 . In the standard testing arm, the proportion of households that reported boiling their drinking water in the previous two weeks increased from 0.03 to 0.45 . This is the equivalent to a 0.38 relative change in a respondent reporting boiling at end line compared to the messaging-only arm after adjusting for baseline characteristics $(\mathrm{p}<0.01)$. In the test kit arm, the percentage of households that reported boiling their drinking water in the previous two weeks rose from 0.02 to 0.34 ; equivalent to a 0.27 relative change compared to the messaging-only arm $(\mathrm{p}<0.01)$. 
There was little change in the proportion of households that reported using a commercial water filter in the previous two weeks. In the standard testing arm, the percentage of households that reported using a commercial water filter remained constant at $1 \%$ between baseline and follow up. In the test kit arm, the proportion of households decreased from $2 \%$ to less than $1 \%$. Among households in the messaging-only arm, the proportion remained constant at less than $1 \%$.

Among all three study arms, the proportion of households that reported using a covered storage container for their household drinking water, as well as the proportion that had soap available at their handwashing station, increased. For households in the standard testing arm, the proportion of households that reported using a covered water container increased from 0.96 to 0.98, but improvement was less than what was observed in the messaging-only arm $(\mathrm{p}=0.07)$. In addition, the proportion of households with soap available at their handwashing station increased from 0.94 to 0.97 , though again this was less than the improvement observed in the messagingonly $\operatorname{arm}(\mathrm{p}=0.05)$.

Among households in the test kit arm, the proportion of households using a covered water storage container increased from 0.93 to 1.0 . The proportion of households with soap available for handwashing increased from 0.89 to 0.99 . Neither change was significant compared to the messaging-only arm $(\mathrm{p}=0.21$ and $\mathrm{p}=0.36$, respectively).

The proportion of households that reported at least one case of diarrhea in the 7 days prior to the survey decreased by a large amount in all three treatment groups. However, improvements in the test kit arm and standard testing arm were not statistically significant compared to the messaging-only arm $(\mathrm{p}=0.59$ and $\mathrm{p}=0.51$, respectively). 
Table 2. Differences in water quality and key behaviors between treatment cohorts and messaging-only group.

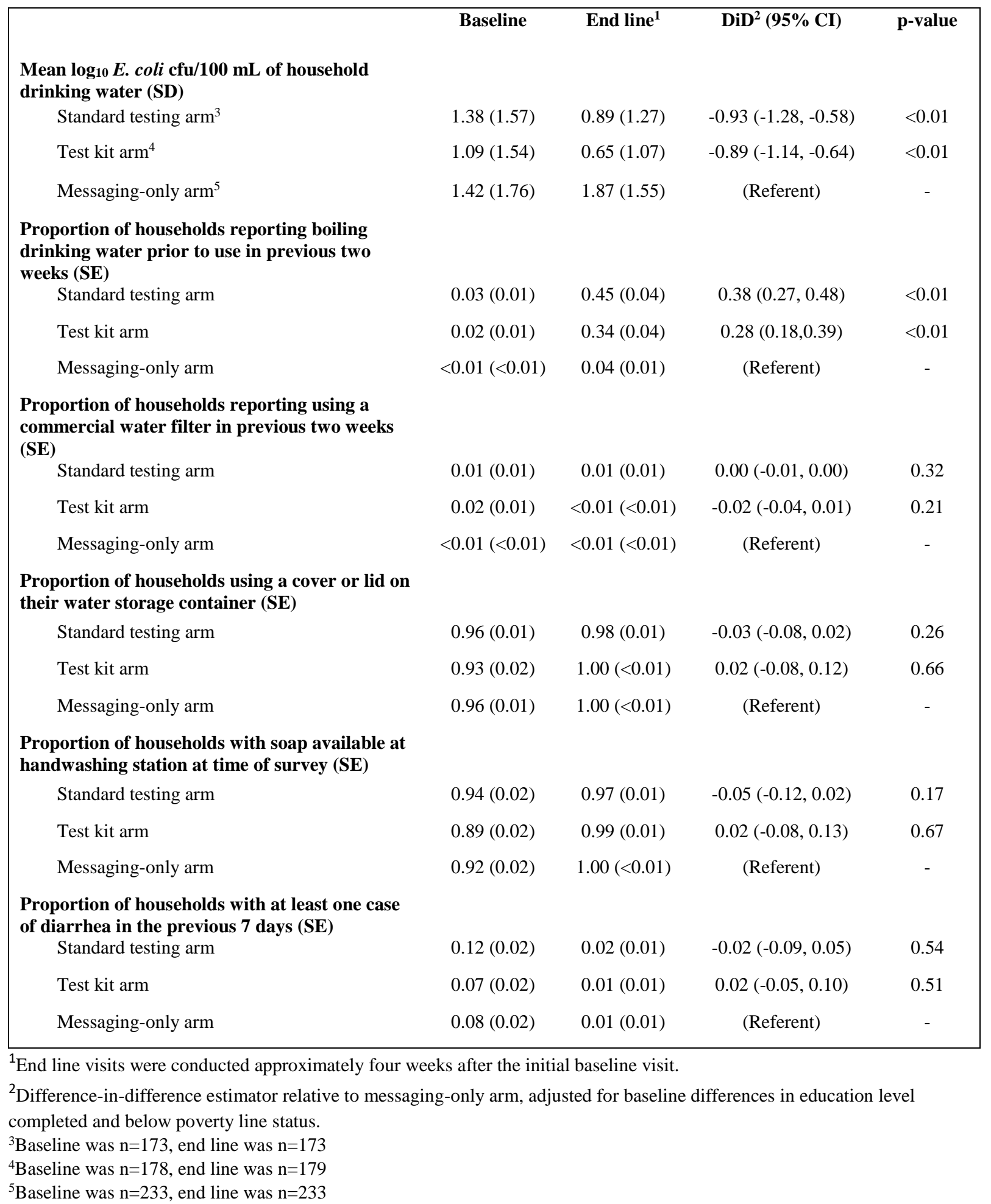


372

373

374

375

376

377

378

379

380

381

382

383

384

385

386

387

388

389

390

391

392

393

\section{Contamination Signal}

Table 3 compares changes in water quality and self-reported water management behaviors between households that received contamination signals and those that did not in both the standard testing arm and the test kit arm. As this analysis breaks the primary study randomization, results should be interpreted with caution.

Standard testing arm

Eighty four percent of households in the standard testing arm were informed that their water showed evidence of microbial contamination following baseline data collection. Among households that did not receive a contamination signal, $\log _{10}$ mean E. coli cfu/100 $\mathrm{ml}$ increased from -0.69 to -0.28 . Among households that received a contamination signal, $\log _{10}$ mean E. coli $\mathrm{cfu} / 100 \mathrm{ml}$ decreased from 1.78 to 1.13 , which corresponds to a 1.08 reduction compared to the households which did not receive a contamination signal $(\mathrm{p}<0.01)$.

Among households in the standard testing arm that did not receive a contamination signal, the proportion that reported boiling their drinking water in the previous two weeks increased from 0 to 0.15 . Among households in the standard testing arm that received a contamination signal, the proportion of households that reported boiling their drinking water increased from 0.04 to 0.50 , which corresponds to a 0.31 relative change compared to households that did not receive a contamination signal $(\mathrm{p}<0.01)$.

Test kit arm

All households in the test kit arm reported using at least two of the provided test kits. The mean number of reported test kits used was 5.9. Among households in the test kit arm, $38 \%$ percent reported at least one test kit yielding a positive result (contamination signal). 
Among households in the test kit arm that did not receive a contamination signal, $\log _{10}$

395 mean E. coli $\mathrm{cfu} / 100 \mathrm{ml}$ increased from 0.22 to 0.24 . Among households that received a 396 contamination signal, $\log _{10}$ mean $E$. coli $\mathrm{cfu} / 100 \mathrm{ml}$ decreased from 2.50 to 1.25 , corresponding to 397 a 1.25 reduction compared to the households that did not receive a contamination signal $(\mathrm{p}<0.01)$. Among households in the test kit arm that did not receive a contamination signal, the 399 proportion that reported boiling their drinking water in the previous two weeks increased from $400 \quad 0.02$ to 0.15 . Among households in the test kit arm that received a contamination signal, the 401 proportion of households that reported boiling their drinking water increased from 0.02 to 0.67 , 402 which corresponds to a 0.53 relative change compared to households that did not receive a 403 contamination signal $(\mathrm{p}<0.01)$. 
Table 3. Difference-in-difference analysis* of water quality and reported water treatment between households that received a contamination signal and households that did not receive a contamination signal

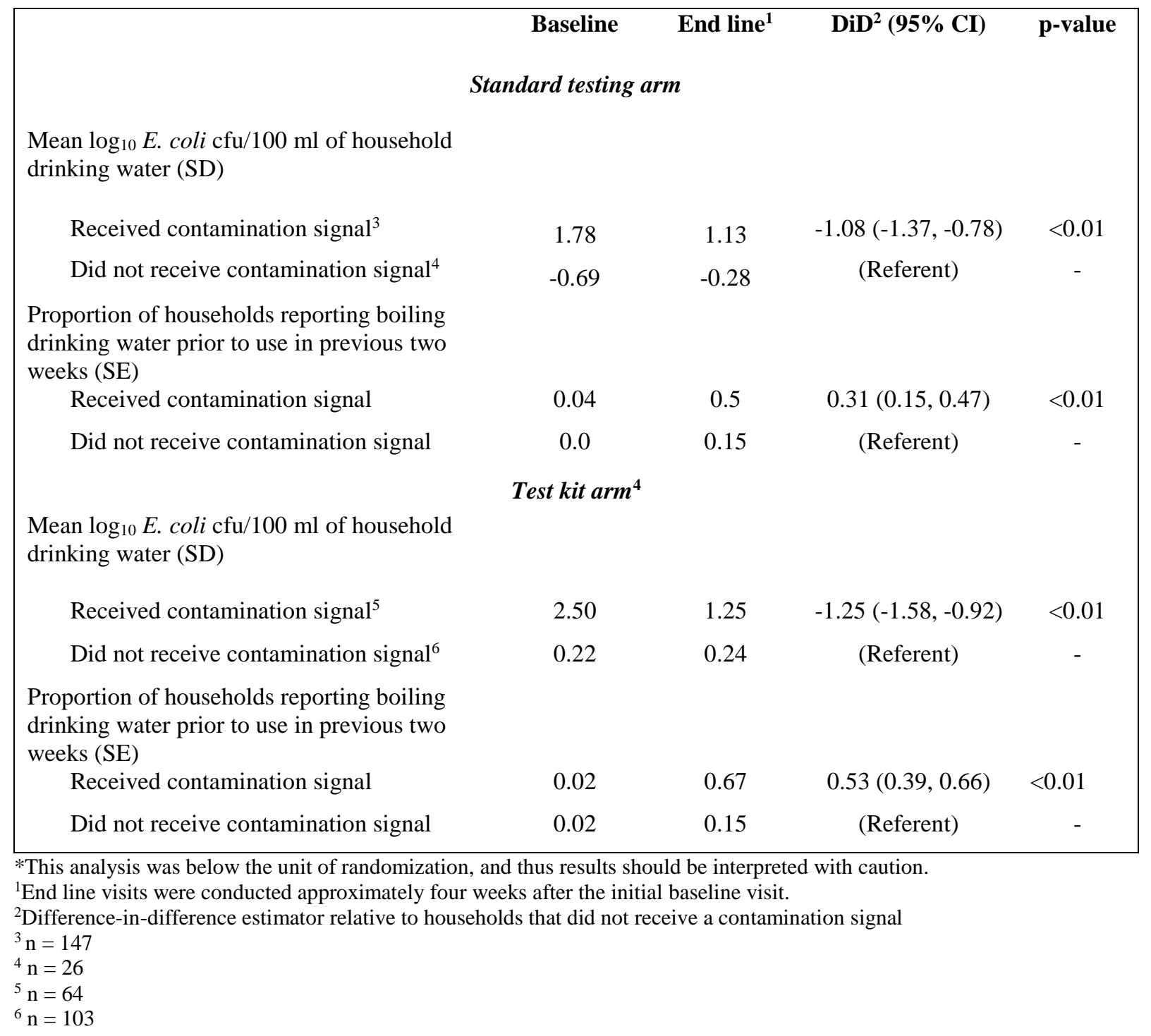

412 Discussion

413 In this study, we explored the effectiveness of using low-cost, field-deployable 414 microbiological water test kits as informational interventions to trigger household-level water 415 management behaviors intended to increase water quality. We found that when given household416 specific information about their drinking water quality, participants were more likely to report 417 boiling their drinking water at the point-of-use and to have safer water overall as indicated by $E$. 
418 coli counts in household drinking water after a four-week follow up period. We detected no

419 significant difference in these outcomes between intervention arms, suggesting that both one-time

420 laboratory reports or user-obtained semi-quantitative household test data, when combined with

421 basic water management messaging, can result in lower short-term counts of $E$. coli in household

422 drinking water compared with messaging only. We found that changes to drinking water quality

423 were consistent with self-reported changes to behavior and that households receiving information

424 indicating baseline water quality was impaired were more likely to take action to improve water

425 safety.

426 Behavior change findings are consistent with previous studies in similar populations in

427 India. In a Delhi suburb, Jalan and Somanathan ${ }^{46}$ utilized a rapid presence/absence fecal indicator

428 test to inform households whether their drinking water was likely to be contaminated, in addition

429 to providing information on available water purification strategies. Intervention households that

430 were informed their water was contaminated were $11 \%$ more likely to adopt a purification strategy

431 after 8 weeks than households that received only information on available purification strategies.

432 Hamoudi et $\mathrm{al}^{47}$ tested a similar intervention in Andhra Pradesh, India, and found that households

433 that received rapid fecal indicator test results and a list of strategies for preventing contamination

434 were more likely to switch to a community-level commercial water source that was available in

435 most study villages, compared to households that received no test results or information. However,

436 the specific changes in behaviors varied as a function of available options - switching of sources

437 or greater household treatment using boiling, or in the case of the Delhi study, filtering - across

438 these studies.

A randomized trial in Ghana ${ }^{48}$ also found the provision of household water quality testing and information to be effective in triggering safe water management behaviors. However, this 
441 study differed from ours in that households did not receive individualized visits. Rather, members

442 of the communities were randomly selected to participate in group workshops tailored for either

443 adults or school children, after which they received test kits to use at their own discretion. Demand

444 for the water test kits was relatively high, as approximately $50 \%$ of recruited adults and $79 \%$ of 445 recruited children chose to attend the two-day workshops. Both treatment groups saw 446 improvements in safe water management behaviors compared to the comparison group that 447 received no information or testing supplies.

448 Research in other settings has not always found information provision to be effective ${ }^{21,41}$.

449 For example, Davis et $\mathrm{al}^{41}$ conducted a study in Dar es Salaam, Tanzania in which households 450 were divided into four groups. The information-only group received educational messaging on 451 how to reduce the risk of waterborne disease. This messaging was also given to the three 452 intervention groups, in addition to the results of household water quality and/or hand-rinse tests. 453 However, there were no significant improvements in water quality among the treatment groups 454 compared to the control households.

Although the majority of households in our study were using an "improved" water source, 456 nearly $80 \%$ of drinking water samples at baseline had evidence of contamination. This was 457 unsurprising, as previous studies have found that "improved" water sources in low- and middle458 income settings frequently have evidence of contamination ${ }^{10,49}$. Thus, point-of-use treatment and 459 safe water management strategies may have an important role to play in mitigating exposure to 460 enteric pathogens in India. Studies in rural Indian populations suggest that point-of-use water 461 treatment methods, such as boiling, solar disinfection, and chlorination are effective in improving 462 water quality, but uptake of these practices is $1 \mathrm{w}^{12,50-53}$. In our study population, only $3 \%$ of 463 participants reported ever treating their drinking water at baseline. This increased significantly 
464 among households that received household-specific water quality information. Although longterm effects on behavior and water quality were beyond the scope of this study, results in the short466 term are promising and warrant further research. laboratory confirmed samples. This could be due to difference in sampling times in the household,

469 differential recall, or different sensitivity in test. It is also possible that participants in the test kit 470 arm used the test kits on samples other than stored household drinking water, such as samples from

471 source water. We did not compare E. coli detection via membrane filtration versus the test kits in

472 duplicate samples; participants tested water separately and reported results back to us up to a month

473 later. However, report of a contamination signal was associated with higher self-reported adoption

474 of safe water management behaviors and greater improvements in household water quality.

Diarrhea prevalence was a tertiary outcome measure for our study; we did not calculate 476 sample size to detect an effect of either intervention on diarrheal prevalence. Low prevalence of 477 diarrhea in the study population ultimately precluded detection of any potential effect on this 478 outcome. We also observed a decrease in diarrhea prevalence in the messaging-only arm between 479 baseline and end line, but there was an increase in E. coli concentration in this study arm over the 480 same time period. We hypothesize that these changes could reflect inherent variability or seasonal 481 effects ${ }^{54}$. 484 improve households' ability to improve and maintain the quality of their own water. However, in 485 the absence of a specific contamination signal - and, in turn, a change in perceived susceptibility 
486 - behavior change was limited. Information alone may result in only limited adoption of water 487 management behaviors unless strategies are in place to turn abstract information about water 488 quality into specific and actionable information.

Unfortunately, water quality testing via current standard laboratory-based methods is not scalable in many settings, including in India, where the requisite trained staff, specialized equipment, basic laboratory infrastructure, and costly consumables may not be widely available outside major cities. According to Government of India estimates covering the rural population only (920 million people), there are 2281 water testing laboratories serving 1.1 million public and private water supplies; of these, a subset regularly test water supplies for microbial contamination. Of 476 laboratories reporting availability of specific tests, $223(57 \%)$ list capacity for basic water microbial parameters (including E. coli specifically). ${ }^{55}$ An estimated 2.24 million water quality tests (any parameters) were conducted in the fiscal year ending in October 2017. Overall, availability of water testing data is very limited throughout the country. Where testing exists, results may not be readily available to consumers, partly because of logistical barriers to re-visiting communities to communicate results. Under these constraints, consumer self-testing, through models such as the test kit, may represent a compelling alternative and allow for scaling up water quality information access to more people at lower cost.

\section{Limitations}

This study had a number of important limitations. First, the short, one-month timeline precludes any assessment of the long-term effects of the interventions. Ideally, changes in behavior can be sustained over time, but they may fade, and future studies should evaluate the longevity of effects as well as the potential benefits of ongoing testing, either by outside actors or by households themselves. A recent systematic review of behavioral impacts of sanitation and hygiene 
interventions suggest that interventions that focus on education and information alone often result in short-term improvements in hygiene behaviors but are likely ineffective at ensuring longer-term sustained change ${ }^{56}$. However, the authors noted that interventions going beyond simple messaging

512 and are grounded in psychological or social theory - such as the EPPM model which informed our 513 intervention development - are associated with increased adherence and sustainability of behavior 514 changes, although data are limited. Second, since we based random selection of households on 515 participatory mapping from village leaders, it is possible this introduced bias toward households 516 or areas of the village that the leader prioritized, resulting in a biased sample. Maps clearly defining 517 village boundaries were unavailable; we considered our approach the best available option. 518 Because mapping used similar processes across all study arms, any selection bias introduced 519 through this system is likely to have been non-differential. Third, though water quality was based 520 on objective measures, data on household behaviors and health outcomes were self-reported. Self521 report for water management and treatment behaviors may be biased, with respondents potentially 522 over-reporting safe behaviors ${ }^{57-59}$. Over-reporting due to courtesy bias, social desirability bias, or 523 other biases may be increased when respondents have been primed (during the intervention) with 524 information about safe water management and treatment behaviors. The survey team administering 525 the end line questionnaire were the same individuals who also provided the messaging component 526 that all study groups received. Self-report bias, if present, would be expected to affect all study 527 arms. Further, observed changes in water quality were consistent with changes in self-reported 528 behaviors within the study population. Finally, in our study, test kits that were used and interpreted 529 by household members had a similar impact on household water quality compared to standard lab 530 testing. However, we note that households in the test kit arm still received household visits and 531 information sessions. The potential effects and cost-effectiveness of these kits or other types of 
532 self-testing when purchased commercially or distributed at the community level - without a

533 substantial messaging component - warrants further investigation.

534 Findings from this study suggest that the provision of household-specific water quality 535 information, when coupled with education and information on low-cost water management 536 strategies, can result in improved water management behaviors and improved water quality. 537 However, changes in behavior may be dependent on whether testing data indicate water is unsafe, 538 and therefore whether action is required to improve water quality. Low cost water quality test kits 539 can provide a possible means of both informing households of their own water quality and 540 providing them with resources to test multiple sources or at multiple points in time, generating 541 actionable feedback on household water management. This allows consumers to determine for 542 themselves whether water is safe and to decide on appropriate measures for protecting the 543 household's drinking water quality. Future studies should focus on whether the changes we 544 observed can be replicated in other settings and extended over longer-term periods, given the 545 challenges of achieving sustained behavior change.

\section{Acknowledgements}

547 We thank the data collection team at the Indian Institute of Technology Kanpur, including 548 Shivshankar Mishraa and Harishankar, for their excellent work. We gratefully acknowledge 549 funding support from the Global Innovation Initiative of Institute of International Education (IIE), 550 through grant 222GAT9817 to the Georgia Tech Research Corporation. We thank Biosynth AG 551 and Thermo Fisher Scientific for preparing and donating chromogenic media used in microbial 552 test kits. 


\section{References}

1. Troeger C, Forouzanfar M, Rao PC, et al. Estimates of global, regional, and national morbidity, mortality, and aetiologies of diarrhoeal diseases: a systematic analysis for the Global Burden of Disease Study 2015. The Lancet Infectious Diseases.17(9):909-948.

2. Lakshminarayanan S, Jayalakshmy R. Diarrheal diseases among children in India: Current scenario and future perspectives. Journal of Natural Science, Biology, and Medicine. 2015;6(1):24-28.

3. Wagner EG, Lanoix JN. Excreta disposal for rural areas and small communities. Monograph series World Health Organization. 1958;39:1-182.

4. Prüss-Ustün A, Bartram J, Clasen $T$, et al. Burden of disease from inadequate water, sanitation and hygiene in low- and middle-income settings: a retrospective analysis of data from 145 countries. Tropical Medicine \& International Health. 2014;19(8):894-905.

5. Wolf J, Prüss-Ustün A, Cumming $O$, et al. Systematic review: Assessing the impact of drinking water and sanitation on diarrhoeal disease in low- and middle-income settings: systematic review and meta-regression. Tropical Medicine \& International Health. 2014;19(8):928-942.

6. Whittington $D$, Hanemann M, Sadoff C, Jeuland M. The Challenge of Improving Water and Sanitation Services in Less Developed Countries. Vol 42008.

7. WHO/UNICEF. Progress on drinking water, sanitation and hygiene: 2017 update and SDG baselines. 2017.

8. Onda K, LoBuglio J, Bartram J. Global Access to Safe Water: Accounting for Water Quality and the Resulting Impact on MDG Progress. International Journal of Environmental Research and Public Health. 2012;9(3):880-894.

9. Bain R, Cronk R, Hossain R, et al. Global assessment of exposure to faecal contamination through drinking water based on a systematic review. Tropical Medicine \& International Health. 2014;19(8):917-927.

10. Bain R, Cronk R, Wright J, Yang H, Slaymaker T, Bartram J. Fecal Contamination of DrinkingWater in Low- and Middle-Income Countries: A Systematic Review and Meta-Analysis. PLOS Medicine. 2014;11(5):e1001644.

11. Waddington $\mathrm{H}$, Snilstveit $\mathrm{B}$, White $\mathrm{H}$, Fewtrell $\mathrm{L}$. Water, sanitation and hygiene interventions to combat childhood diarrhoea in developing countries. Vol 12009.

12. Thompson T, Sobsey M, Bartram J. Providing clean water, keeping water clean: an integrated approach. International Journal of Environmental Health Research. 2003;13(sup1):S89-S94.

13. Rosa G, Clasen T. Estimating the Scope of Household Water Treatment in Low- and MediumIncome Countries. The American Journal of Tropical Medicine and Hygiene. 2010;82(2):289-300.

14. Luoto J, Najnin N, Mahmud M, et al. What point-of-use water treatment products do consumers use? Evidence from a randomized controlled trial among the urban poor in Bangladesh. PLOS One. 2011;6(10):e26132.

15. Brown J, Clasen T. High Adherence Is Necessary to Realize Health Gains from Water Quality Interventions. PLOS ONE. 2012;7(5):e36735.

16. Luoto J, Mahmud M, Albert J, et al. Learning to Dislike Safe Water Products: Results from a Randomized Controlled Trial of the Effects of Direct and Peer Experience on Willingness to Pay. Environmental Science \& Technology. 2012;46(11):6244-6251.

17. Solawetz Hulland K, Martin N, Dreibelbis R, DeBruiker Valliant J, Winch P. What factors affect sustained adoption of safe water, hygiene and sanitation technologies? A systematic review of literature. 2015.

18. Figueroa M.E., Kincaid D. Social, Cultural and Behavioral Correlates of Household Water Treatment and Storage. Center Publication HCl 2010-1: Health Communication Insights, 
Baltimore: Johns Hopkins Bloomberg School of Public Health, Center for Communication Programs. 2010.

19. Jeuland M, Orgill J, Shaheed A, Revell G, Brown J. A matter of good taste: investigating preferences for in-house water treatment in peri-urban communities in Cambodia. Environment and Development Economics. 2015;21(3):291-317.

20. Whittington D, Jeuland M, Barker K, Yuen Y. Setting Priorities, Targeting Subsidies among Water, Sanitation, and Preventive Health Interventions in Developing Countries. Vol 402012.

21. Brown J, Hamoudi A, Jeuland M, Turrini G. Seeing, believing, and behaving: Heterogeneous effects of an information intervention on household water treatment. Journal of Environmental Economics and Management. 2016.

22. Bennear L, Tarozzi A, Pfaff A, Balasubramanya S, Ahmed KM, van Geen A. Impact of a Randomized Controlled Trial in Arsenic Risk Communication on Household Water-Source Choices in Bangladesh. Journal of environmental economics and management. 2013;65(2):225240.

23. Madajewicz M, Pfaff A, van Geen A, et al. Can information alone change behavior? Response to arsenic contamination of groundwater in Bangladesh. Journal of Development Economics. 2007;84(2):731-754.

24. Troeger C, Forouzanfar M, Rao PC, et al. Estimates of global, regional, and national morbidity, mortality, and aetiologies of diarrhoeal diseases: a systematic analysis for the Global Burden of Disease Study 2015. The Lancet Infectious Diseases.

25. Ercumen A, Arnold BF, Kumpel E, et al. Upgrading a piped water supply from intermittent to continuous delivery and association with waterborne illness: a matched cohort study in urban India. PLoS Med. 2015;12(10):e1001892.

26. Bivins AW, Sumner T, Kumpel E, et al. Estimating Infection Risks and the Global Burden of Diarrheal Disease Attributable to Intermittent Water Supply Using QMRA. Environ Sci Technol. 2017;51(13):7542-7551.

27. Hayes RaM, L.H. . Cluster Randomized Controlled Trials. New York: Chapman \& Hall/CRC Press; 2009.

28. Haas CN. How to average microbial densities to characterize risk. Water Research. 1996;30(4):1036-1038.

29. Arnold BF, Galiani S, Ram PK, et al. Optimal recall period for caregiver-reported illness in risk factor and intervention studies: a multicountry study. American journal of epidemiology. 2013;177(4):361-370.

30. Kanpur Dehat Village and Townwise Primary Census Abstract. Census of India 2011. 2011.

31. Bain RE, Woodall C, Elliott J, et al. Evaluation of an Inexpensive Growth Medium for Direct Detection of Escherichia coli in Temperate and Sub-Tropical Waters. PLoS One. 2015;10(10):e0140997.

32. Magro G, Bain RE, Woodall CA, Matthews RL, Gundry SW, Davis AP. Synthesis and application of resorufin beta-D-glucuronide, a low-cost chromogenic substrate for detecting Escherichia coli in drinking water. Environ Sci Technol. 2014;48(16):9624-9631.

33. Brown J, Stauber C, Murphy JL, et al. Ambient-temperature incubation for the field detection of Escherichia coli in drinking water. Journal of applied microbiology. 2011;110(4):915-923.

34. Witte K. Putting the fear back into fear appeals: The extended parallel process model. Communications Monographs. 1992;59(4):319-326.

35. Ray I. Women, Water, and Development. Annual Review of Environment and Resources. 2007;32(1):421-449.

36. Hayes RJ, Bennett S. Simple sample size calculation for cluster-randomized trials. International Journal of Epidemiology. 1999;28(2):319-326. 
37. Duflo E, Glennerster R, Kremer M. Using Randomization in Development Economics Research: A Toolkit. T. Schultz and John Strauss, eds., Handbook of Development Economics. Vol 4. Amsterdam and New York: North Holland; 2008.

38. Fleiss JL. Appendix: Sample-Size Determination. The Design and Analysis of Clinical Experiments: John Wiley \& Sons, Inc.; 1999:369-417.

39. Moher D, Schulz KF, Altman DG. The CONSORT statement: revised recommendations for improving the quality of reports of parallel-group randomised trials. The Lancet. 2001;357(9263):1191-1194.

40. Agency USEP. Method 1604: Total coliforms and Escherichia coli in water by membrane filtration using a simultaneous detection technique (MI Medium). Vol Publication EPA-821-R-02-024. USEPA Office of Water (4303T), Washington, D.C.2002.

41. Davis J, Pickering AJ, Rogers K, Mamuya S, Boehm AB. The Effects of Informational Interventions on Household Water Management, Hygiene Behaviors, Stored Drinking Water Quality, and Hand Contamination in Peri-Urban Tanzania. The American Journal of Tropical Medicine and Hygiene. 2011;84(2):184-191.

42. Zhou H, Taber C, Arcona S, Li Y. Difference-in-Differences Method in Comparative Effectiveness Research: Utility with Unbalanced Groups. Applied Health Economics and Health Policy. 2016;14(4):419-429.

43. Zeger SL, Liang KY. Longitudinal data analysis for discrete and continuous outcomes. Biometrics. 1986;42(1):121-130.

44. Little RJAaR, D.B. Statistical Analysis with Missing Data, Second Editio. Hoboken, NJ: John Wiley and Sons; 2002.

45. WHO. Guidelines for Drinking-water Quality, Fourth Edition. 2017.

46. Jalan J, Somanathan E. The importance of being informed: Experimental evidence on demand for environmental quality. Journal of Development Economics. 2008;87(1):14-28.

47. Hamoudi A, Jeuland M, Lombardo S, Patil S, Pattanayak SK, Rai S. The effect of water quality testing on household behavior: evidence from an experiment in rural India. Am J Trop Med Hyg. 2012;87(1):18-22.

48. Okyere CYP, Evita Hanie; Asante, Felix Ankomah; von Braun, Joachim. The Impacts of Household Water Quality Testing and Information on Safe Water Behaviors: Evidence from a Randomized Experiment in Ghana. ZEF- Discussion Papers on Development Policy No 234. 2017.

49. Shaheed A, Orgill J, Montgomery MA, Jeuland MA, Brown J. Why "improved" water sources are not always safe. Bulletin of the World Health Organization. 2014;92(4):283-289.

50. Clasen T, McLaughlin C, Nayaar N, et al. Microbiological Effectiveness and Cost of Disinfecting Water by Boiling in Semi-urban India. The American Journal of Tropical Medicine and Hygiene. 2008;79(3):407-413.

51. Rose A, Roy S, Abraham V, Holmgren G, George K, Balraj V. Solar disinfection of water for diarrhoeal prevention in southern India. Arch Dis Child. 2006;91.

52. Kattula D, Francis MR, Kulinkina A, et al. Environmental predictors of diarrhoeal infection for rural and urban communities in south India in children and adults. Epidemiology and Infection. 2015;143(14):3036-3047.

53. Boisson S, Stevenson M, Shapiro L, et al. Effect of Household-Based Drinking Water Chlorination on Diarrhoea among Children under Five in Orissa, India: A Double-Blind Randomised PlaceboControlled Trial. PLoS Medicine. 2013;10(8):e1001497.

54. Kulinkina AV, Mohan VR, Francis MR, et al. Seasonality of water quality and diarrheal disease counts in urban and rural settings in south India. Scientific reports. 2016;6:20521.

55. India Go. Ministry of Drinking Water and Sanitation: National Rural Drinking Water Programme. 2017; indiawater.gov.in. Accessed 31 October 2017, 2017. 
56. De Buck E VRH, Hannes K, Govender T, Naidoo S, Avau B,, Vande veegaete A MA, Vittoria L, Cargo M, Mosler H-J,, Vandekerckhove P YT. Approaches to promote handwashing and sanitation behaviour change in low- and middle-income countries: a mixed method systematic review. . Campbell Systematic Reviews. 2017;7.

57. Rosa G, Clasen T. Consistency of Use and Effectiveness of Household Water Treatment among Indian Households Claiming to Treat Their Water. The American Journal of Tropical Medicine and Hygiene. 2017;97(1):259-270.

58. Brown J, Sobsey MD. Boiling as household water treatment in Cambodia: a longitudinal study of boiling practice and microbiological effectiveness. Am J Trop Med Hyg. 2012;87(3):394-398.

59. Psutka R, Peletz R, Michelo S, Kelly P, Clasen T. Assessing the microbiological performance and potential cost of boiling drinking water in urban Zambia. Environ Sci Technol. 2011;45(14):60956101. 